\title{
Modified Spatially Adaptive Denoising Algorithm for an Image Corrupted by Gaussian Noise
}

\author{
Arabinda Dash $^{1}$, Sujaya Kumar Sathua ${ }^{2}$ \\ M. Tech Scholar, Dept., of CSE \& IT, Veer Surendra Sai University of Technology, Burla, Sambalpur, Odisha, India ${ }^{1}$ \\ Assistant Professor, Dept. of CSE \& IT, Veer Surendra Sai University of Technology, Burla, Sambalpur, Odisha, India ${ }^{2}$
}

\begin{abstract}
A modified spatially adaptive denoising algorithm for a single image corrupted by Gaussian noise is proposed in this paper. The proposed algorithm use local statistics of a selected window i.e. by defining local weighted mean, local weighted activity and local maximum. These local statistics are used to detect the noise in the image then a modified Gaussian filter is used for noise suppression. This algorithm is tested against different images and the experimental result shows its result is better than different existing methods like Pixel Wise Median Absolute Difference (PWMAD), Rank Order Criteria (ROC), Switching-based Adaptive Weighted Mean (SAWM) and Spatially Adaptive Denoising Algorithm (SADA).
\end{abstract}

Keywords: Gaussian Noise, Denoising, Local statistics and Gaussian filter.

\section{INTRODUCTION}

Gaussian noise is present in image due to the additive noise caused by noise sensor, recording process, communication channels and any combination of them [1]. In Gaussian noise the Probability Density Function (PDF) is equal to the normal distribution. Noise removal is very important for subsequent processing operation because the captured image by the imaging system represents the degraded version of an original image due to additive noise. Therefore we can say that Image Restoration plays an important role in Digital Image Processing. There are many algorithms has been developed for the noise removal over past two decades. Among these algorithms, generally mean filters, median filters and their modified methods are used due to their good noise removal capacity and low computational cost benefits[2]-[7].

There are many algorithms like Pixel-Wise Median absolute Difference (PWMAD) [8], Rank Order Criteria (ROC) [9], and Switching-based Adaptive Weighted Mean (SAWM) filter [10] are uses a noise detection stage in order to achieve better results. The noise detection approaches in these algorithms are different like in ROC, the signal samples are compared within a narrow rank window to detect the noisy pixels, whereas in SAWM, the noisy pixels are detected by comparing the minimum absolute value of four mean differences between the current pixel and its neighbors in four directional windows with a pre-defined threshold. The above methods still fails to preserve the detail information since the local statistics are not effectively used for the noise removal process.

In order to overcome the drawbacks Spatially Adaptive Denoising Algorithm (SADA) for a single image corrupted by Gaussian noise is proposed [11]. In this method, the noise detection and the noise removal is done by using local statistics of the image. By taking motivation from this SADA method we have proposed a Modified Spatially Adaptive Denoising Algorithm (MSADA), which gives better result than the SADA and other existing algorithms.

This paper is organized as follows: Section II describes the proposed algorithm MSADA. In section III, the experimental and the comparison results will be presented in order to demonstrate the capability of the proposed algorithm. Finally the conclusion is drawn in section IV.

\section{Proposed Algorithm}

In our algorithm denoising of a corrupted image is performed in two processes. First a noise detection process is defined by using the local statistics, such as local weighted mean, local weighted activity and local maximum [11]. Then a spatially adaptive Gaussian filter is used to suppress the detected noise.

When an original image is degraded by additive noise and if the noise is signal independent, then at point $(i, j)$ the degraded image model in two dimensional coordinate can be written as:

$$
x(i, j)=y(i, j)+\eta(i, j)
$$

where $x, y$ and $\eta$ are observed image, original image and noisy image respectively [1]. For an observed pixel $x(i, j)$ the local weighted mean, local weighted activity and local maximum with the window size $(2 \mathrm{U}+1) \times(2 \mathrm{~V}+1)$ are defined as given below:

$$
\begin{array}{r}
\mu(i, j)=\frac{\sum_{m} \sum_{n,(m, n) \in S} w(m, n) x(i+m, j+n)}{\sum_{m} \sum_{n,(m, n) \in S} w(m, n)} \\
\sigma(i, j)=\frac{\sum_{m} \sum_{n,(m, n) \in S} w(m, n)|x(i+m, j+n)-\mu(i, j)|}{\sum_{m} \sum_{n,(m, n) \in S} w(m, n)} \\
x_{\max }(i, j)=\max _{(p, q) \in S} x(p, q)
\end{array}
$$

where, $\mu(i, j)$ is local weighted mean, $\sigma(i, j)$ is local weighted activity, $x_{\max }(i, j)$ is local maximum, $w(m, n)$ is the weighting coefficient at point $(m, n)$ in a selected 
window of region $\mathrm{S}$. In this work, $\mathrm{m}$ is from $-\mathrm{U}$ to $\mathrm{U}$ and $\mathrm{n}$ Step- 5: Repeat steps 1 to 4 till the process is complete for is from $-\mathrm{V}$ to $\mathrm{V}$ in the region $\mathrm{S}$. Now the noise detection the entire image.

function using the local statistics in Equations (1), (2) and (3) is defined as given below:

$$
\begin{gathered}
\text { Flag }(i, j)=\left\{\begin{array}{r}
1 \text { if } x(i, j)>\mu(i, j)+B(i, j) \\
\text { or } x(i, j)<\mu(i, j)-B(i, j) \\
0 \quad \text { otherwise }
\end{array}\right. \\
B(i, j)=k \times \frac{\sigma(i, j)}{\operatorname{xmax}(i, j)}
\end{gathered}
$$

where, $k$ represents a constant. When the flag is equal to 1 , then the pixel is detected as corrupted one and the flag is 0 , then the pixel is uncorrupted.

According to HVS [11], when one pixel is detected as corrupted one with lower local weighted activity then strong filtering or over-smoothing process is required, while for an corrupted pixel with higher local weighted activity a weak filtering is required. To control the degree of smoothness of the reconstructed image, Gaussian filtering by using local activity is very useful. Using the local statistics in equations (2) and (3) a modified Gaussian filter is:

$$
h(i, j)=\frac{1}{Z} e^{\left(-T \frac{\sigma(i, j)^{2}\left(i^{2}+j^{2}\right)}{\sqrt{\mu(i, j)}}\right)}
$$

where $z(i, j)$ and $T$ represents the normalizing constant and a tuning parameter respectively. Now by using Gaussian filter the reconstructed pixel for the same region $S$ of the selected window can be written as:

$$
\mathrm{X}(\mathrm{i}, \mathrm{j})=\frac{\sum_{m} \sum_{n,(m, n) \in S} h(i, j) x(i+m, j+n)}{c \times h(i, j)}
$$

where ' $c$ ' represents the total numbers of pixel in the window in which $h(i, j)$ is multiplied. The aim of the proposed algorithm is to detect and remove the noise component effectively by using local statistics from a corrupted image.

\section{Algorithm:}

Modified Spatially Adaptive Denoising Algorithm (MSADA) by using the above equations (2) to (8) of a local window of size $3 \times 3$ i.e. $U=1$ and $V=1$ is given as below:

Step- 1: Select 2-D window of size $3 \times 3$. Assume that the processing pixel as $\mathrm{x}(\mathrm{i}, \mathrm{j})$ which lies at the centre of window.

Step- 2: Find local weighted mean, local weighted activity and local maximum for the processing pixel $x(i, j)$ with in the window.

Step- 3: If $x(i, j)>\mu(i, j)+B(i, j)$ or $x(i, j)<\mu(i, j)-B(i, j)$, Then take flag $(i, j)=1$, the processing pixel is considered to be as corrupted one.

Otherwise flag $(\mathrm{i}, \mathrm{j})=0$, the processing pixel is considered to be as uncorrupted one and it left unchanged.

Step- 4: If flag $(\mathrm{i}, \mathrm{j})=1$, then find the reconstructed pixel $X(i, j)$.
Select a 2 D $3 \times 3$ window with centre element as $x(i, j)$ as a processing pixel

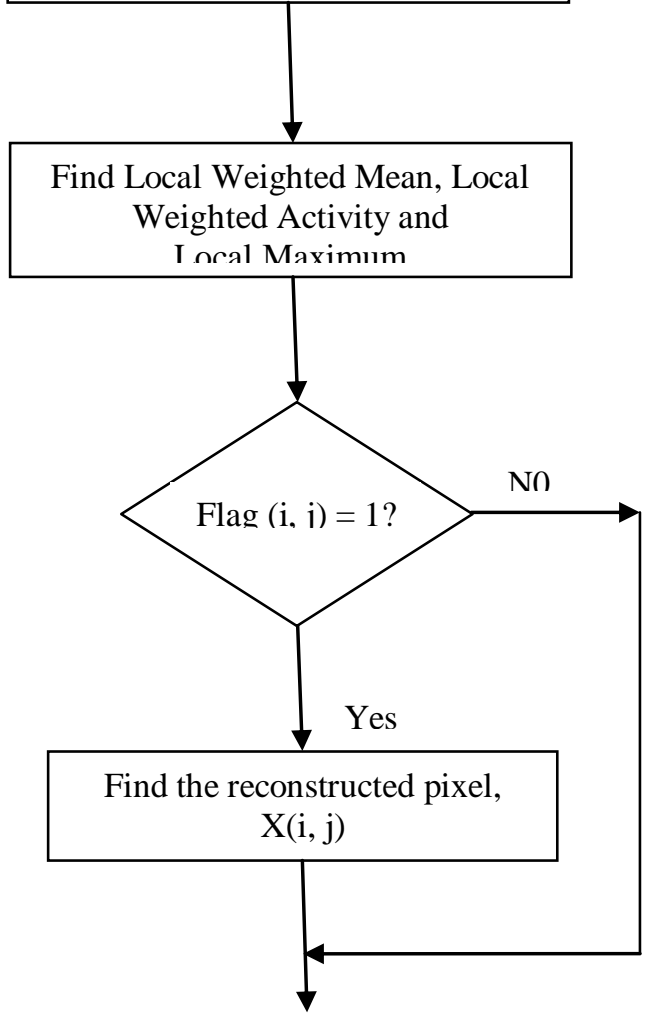

Denoised Image

Fig. 1: Entire procedure of proposed algorithm

\section{EXPERIMENTAL AND COMPARISON RESULTS}

The proposed MSADA algorithm was tested with various images and various SNRs. Gaussian noise is added to the original gray level $256 \times 256$ "Lena.jpg", "Cameraman.tif", and "Goldhill.gif" images. We compared the proposed algorithm with PWMAD, ROC, SAWM and SADA methods.

In our work, $\mathrm{U}=1$ and $\mathrm{V}=1$ are used as to minimize the computational cost and the blurring effect of the reconstructed image, because for larger window size the computational cost and blurring effect is more. The diagonal elements within the window are not used to avoid over smoothness. In addition, the weighting coefficients $\mathrm{w}(1,0)=\mathrm{w}(-1,0)=\mathrm{w}(0,1)=\mathrm{w}(0,-1)=3$, and $\mathrm{w}(0,0)=4$ in equations (2) and (3) are used as to reduced the computational cost by avoiding a division operation.

As $\mathrm{k}$ in equation (6) is higher, the bounds are looser, leading to higher missing detection error. Therefore to minimize the detection error the range of $\mathrm{k}$ is from 0.01 to 0.1 . Now $\mathrm{T}$ in equation (7) is a parameter that determines the smoothing degree. In our work $\mathrm{k}=0.05$ and $\mathrm{T}=0.05$ are used [11]. Lastly $\mathrm{z}$ is a constant that equally applied to 
determine the filter coefficient, therefore $\mathrm{z}=1$ is used as the performance of proposed algorithm doesn't depend on $z$.

By using these above values of $\mathrm{k}, \mathrm{T}$, and $\mathrm{z}$, we have denoise the corrupted image according to the proposed algorithm and the performance is compared with PWMAD, ROC, SAWM, and SADA methods in terms of MSE and PSNR values. The comparison result of MSE and PSNR value for Lena image is shown in Table 1 and 2. The comparison result of MSE and PSNR value for Cameraman image is shown in Table 3 and 4. The comparison result of MSE and PSNR value for Goldhill image is shown in Table 5 and 6. From this comparison it is clear that the proposed algorithm outperforms the other algorithms. In Table 7 the comparison result of IEF value for Lena, Cameraman, and Goldhill images between existing algorithm and SADA. From this comparison it is clear that the existing algorithm have the better result than SADA.

In figure 2 to 7, the performance graph of MSE and PSNR values of proposed algorithm vs. PWMAD, ROC, SAWM and SADA method are shown. And lastly Figure 8 shows enlarged original Lena and image are corrupted with $10 \mathrm{~dB}$ Gaussian noise density and reconstructed image obtained by proposed algorithm and other existing algorithms.

TABLE 1

COMPARISION OF MSE VALUES OF DIFFERENT ALGORITHMS FOR LENA IMAGE AT DIFFERENT NOISE LEVEL

\begin{tabular}{|c|c|c|c|c|c|}
\hline \multirow{2}{*}{$\begin{array}{c}\text { Noise } \\
\text { In } \\
\text { dB }\end{array}$} & \multicolumn{5}{|c|}{ MSE VALUES } \\
\hline & $\begin{array}{c}\text { PWM } \\
\text { AD }\end{array}$ & ROC & SAWM & SADA & PA \\
\hline 10 & 108.7 & 112.0 & 112.9 & 95.4 & 71.15 \\
\hline 20 & 56.1 & 56.0 & 53.1 & 37.8 & 26.86 \\
\hline 30 & 48.9 & 48.2 & 44.3 & 31.9 & 22.28 \\
\hline
\end{tabular}

TABLE 2

COMPARISION OF PSNR VALUES OF DIFFERENT ALGORITHMS FOR LENA IMAGE AT DIFFERENT NOISE LEVEL

\begin{tabular}{|c|c|c|c|c|c|}
\hline \multirow{2}{*}{$\begin{array}{c}\text { Noise } \\
\text { In } \\
\text { dB }\end{array}$} & $\begin{array}{c}\text { PWM } \\
\text { AD }\end{array}$ & ROC & SAWM & SADA & PA \\
\cline { 2 - 6 } & PSNR VALUES \\
\hline 10 & 27.76 & 27.63 & 27.60 & 28.33 & 29.60 \\
\hline 20 & 30.64 & 30.64 & 30.87 & 32.35 & 33.83 \\
\hline 30 & 31.23 & 31.30 & 31.66 & 33.09 & 34.65 \\
\hline
\end{tabular}

TABLE 3

COMPARISION OF MSE VALUES OF DIFFERENT ALGORITHMS FOR CAMERAMAN IMAGE AT DIFFERENT NOISE LEVEL

\begin{tabular}{|c|c|c|c|c|c|}
\hline \multirow{2}{*}{$\begin{array}{c}\text { Noise } \\
\text { In } \\
\text { dB }\end{array}$} & $\begin{array}{c}\text { PWM } \\
\text { AD }\end{array}$ & ROC & $\begin{array}{c}\text { SAW } \\
\text { M }\end{array}$ & SADA & PA \\
\cline { 2 - 6 } & 201.9 & 204.4 & 202.5 & 181.87 & 164.2 \\
\hline 10 & 128.1 & 127.4 & 119.9 & 102.39 & 95.85 \\
\hline 20 & 120.5 & 118.3 & 111.3 & 96.34 & 79.97 \\
\hline
\end{tabular}

Copyright to IARJSET
TABLE 4

COMPARISION OF PSNR VALUES OF DIFFERENT ALGORITHMS FOR CAMERAMAN IMAGE AT DIFFERENT NOISE LEVEL

\begin{tabular}{|c|c|c|c|c|c|}
\hline \multirow{2}{*}{$\begin{array}{c}\text { Noise } \\
\text { In } \\
\text { dB }\end{array}$} & \multicolumn{5}{|c|}{ PSNR VALUES } \\
\cline { 2 - 6 } & $\begin{array}{c}\text { PWMA } \\
\text { D }\end{array}$ & ROC & SAWM & SADA & PA \\
\hline 10 & 25.07 & 25.02 & 25.06 & 25.53 & 25.97 \\
\hline 20 & 27.05 & 27.34 & 27.34 & 28.02 & 28.31 \\
\hline 30 & 27.32 & 27.40 & 27.66 & 28.29 & 29.10 \\
\hline
\end{tabular}

TABLE 5

COMPARISION OF MSE VALUES OF DIFFERENT ALGORITHMS FOR GOLDHILL IMAGE AT DIFFERENT NOISE LEVEL

\begin{tabular}{|c|c|c|c|c|c|}
\hline \multirow{2}{*}{$\begin{array}{c}\text { Noise } \\
\text { In } \\
\text { dB }\end{array}$} & \multicolumn{5}{|c|}{ MSE VALUES } \\
\cline { 2 - 6 } & $\begin{array}{c}\text { PWMA } \\
\text { D }\end{array}$ & ROC & SAWM & SADA & PA \\
\hline 10 & 150.4 & 152.1 & 151.5 & 102.8 & 71.12 \\
\hline 20 & 103.8 & 97.9 & 102.1 & 51.4 & 32.98 \\
\hline 30 & 98.3 & 91.2 & 90.4 & 46.3 & 29.15 \\
\hline
\end{tabular}

TABLE 6

COMPARISION OF PSNR VALUES OF DIFFERENT ALGORITHMS FOR GOLDHILL IMAGE AT DIFFERENT NOISE LEVEL

\begin{tabular}{|c|c|c|c|c|c|}
\hline \multirow{2}{*}{$\begin{array}{c}\text { Noise } \\
\text { In } \\
\text { dB }\end{array}$} & \multicolumn{5}{|c|}{ PSNR VALUES } \\
\cline { 2 - 6 } & $\begin{array}{c}\text { PWM } \\
\text { AD }\end{array}$ & ROC & SAWM & SADA & PA \\
\hline 10 & 26.35 & 26.30 & 26.32 & 28.01 & 29.69 \\
\hline 20 & 27.96 & 28.22 & 28.04 & 31.02 & 32.94 \\
\hline 30 & 28.20 & 28.53 & 28.56 & 31.47 & 33.48 \\
\hline
\end{tabular}

TABLE 7

COMPARISION OF IEF VALUES OF PROPOSED ALGORITH WITH SADA FOR LENA, CAMERAMAN AND GOLDHILL IMAGE AT DIFFERENT NOISE LEVEL

\begin{tabular}{|c|c|c|c|c|c|c|}
\hline \multirow{2}{*}{$\begin{array}{c}\text { Noise } \\
\text { In dB }\end{array}$} & \multicolumn{6}{|c|}{ IEF Values } \\
\cline { 2 - 7 } & Lena Image & \multicolumn{2}{|c|}{$\begin{array}{c}\text { Cameraman } \\
\text { Image }\end{array}$} & \multicolumn{2}{c|}{$\begin{array}{c}\text { Goldhill } \\
\text { Image }\end{array}$} \\
\cline { 2 - 7 } & SADA & PA & SADA & PA & SADA & PA \\
\hline 10 & 136.5 & 176.2 & 93.1 & 108.1 & 181.1 & 209.7 \\
\hline 20 & 429.6 & 465.1 & 152.4 & 175.2 & 409.5 & 451.2 \\
\hline 30 & 533.5 & 559.5 & 158.8 & 187.9 & 392.2 & 510.6 \\
\hline
\end{tabular}

From above comparisons in Table 1 to 7 , it is clear that the proposed algorithm MSADA gives better MSE and PSNR values as compare to other existing algorithms.

From Table 7 it can be seen that the proposed method MSADA also gives better IEF value than the existing algorithm SADA. 


\section{International Advanced Research Journal in Science, Engineering and Technology}

Vol. 2, Issue 3, March 2015

Now the comparison graphs are shown in Figure 2 to 7.

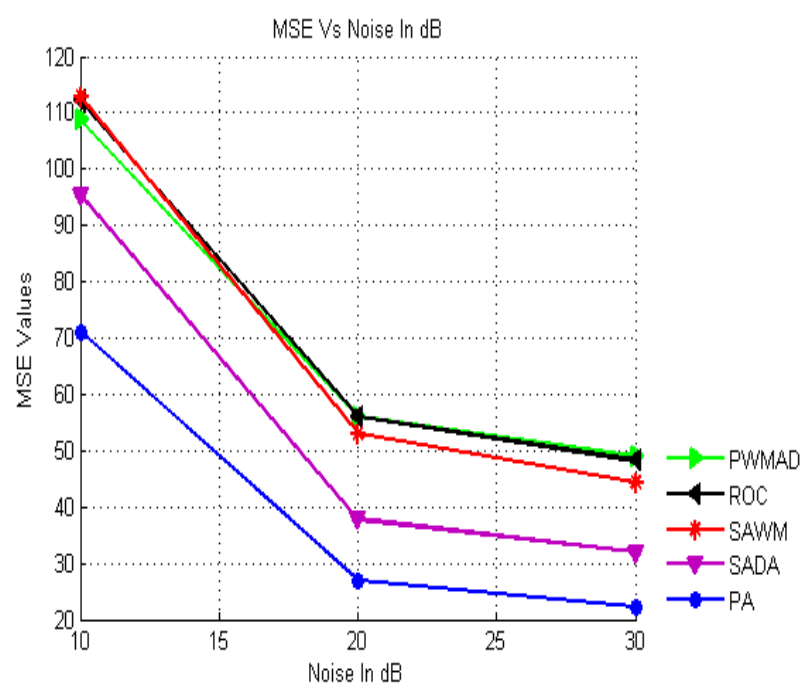

Fig. 2: Comparison graph of MSE values at different noise level in $\mathrm{dB}$ for Lena Image

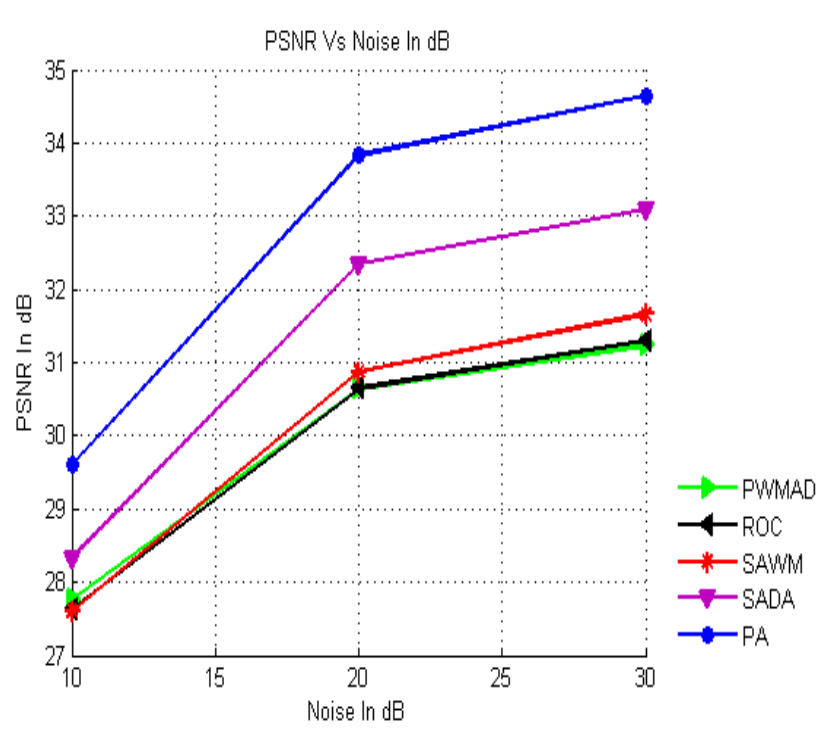

Fig. 3: Comparison graph of PSNR values at different noise level in $\mathrm{dB}$ for Lena Image

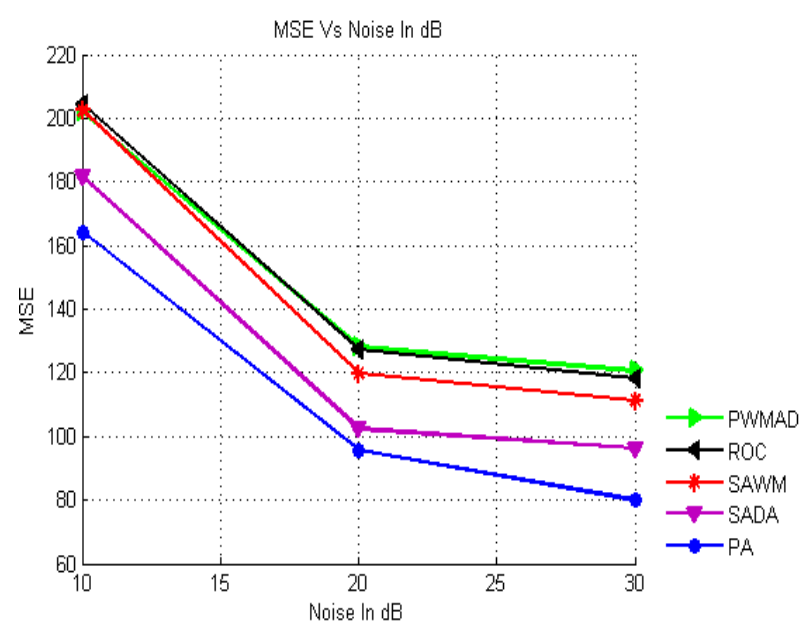

Fig. 4: Comparison graph of MSE values at different noise level in $\mathrm{dB}$ for CAMERAMAN Image

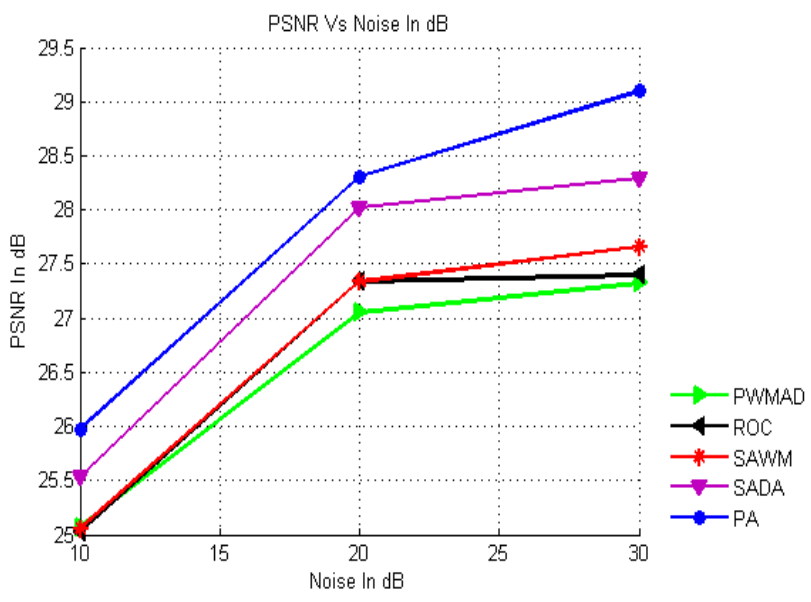

Fig. 5: Comparison graph of PSNR values at different noise level in $\mathrm{dB}$ for CAMERAMAN Image

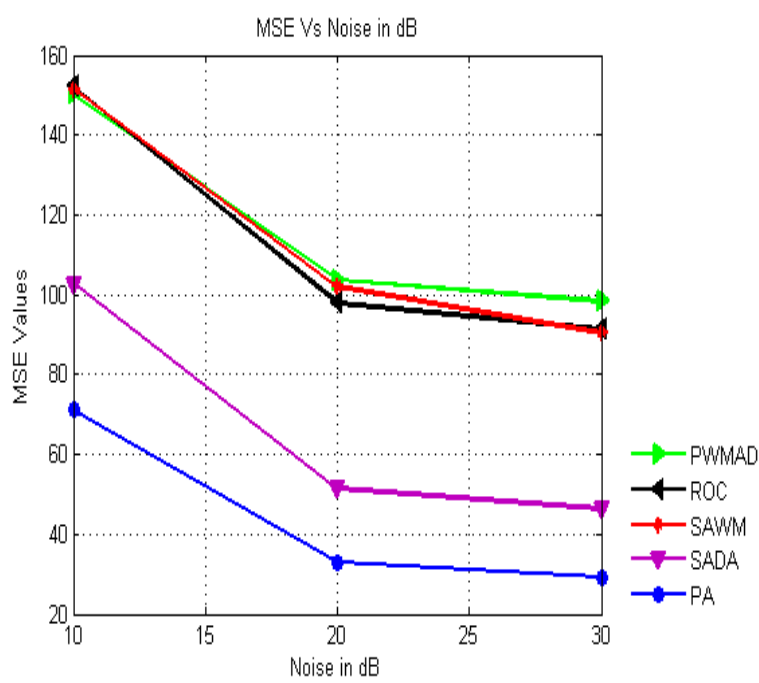

Fig. 6: Comparison graph of MSE values at different noise level in $\mathrm{dB}$ for GOLDHILL Image

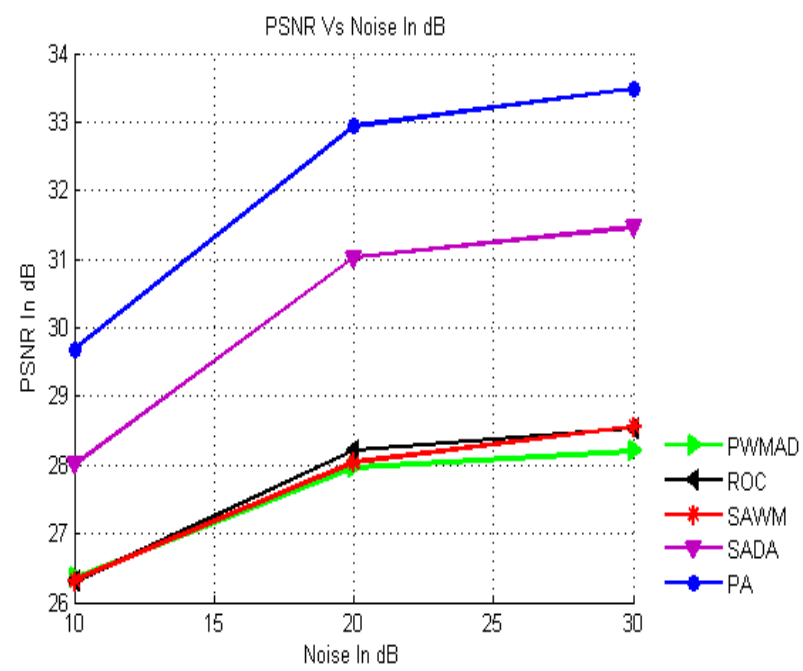

Fig. 7: Comparison graph of PSNR values at different noise level in $\mathrm{dB}$ for GOLDHILL Image 
From the above comparison graph it can seen that the proposed algorithm MSADA perform better than the all existing algorithms.

Now lastly in Figure 8, the enlarged original Lena image is corrupted with $10 \mathrm{~dB}$ Gaussian noise density and reconstructed images obtained by proposed algorithm and other existing algorithms are shown, which is the clear evidence that our proposed algorithm MSADA have the better denoising capability.

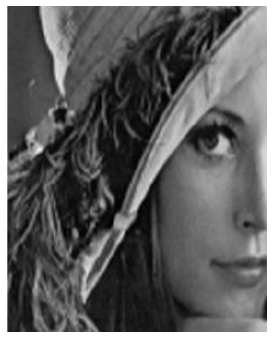

(A)

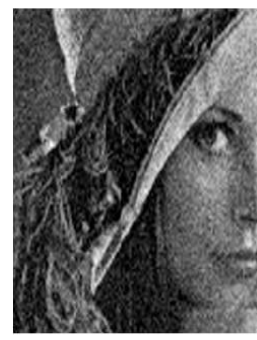

(B)

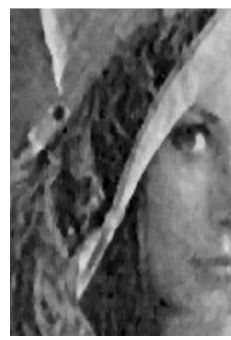

(C)

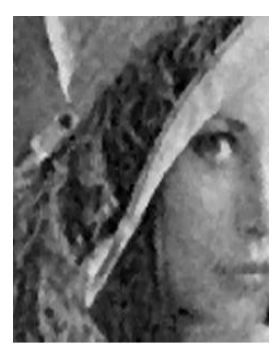

(D)

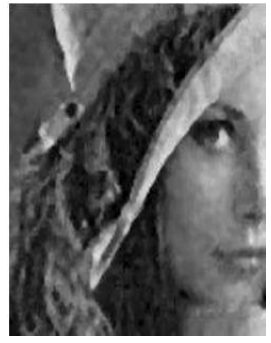

(F)

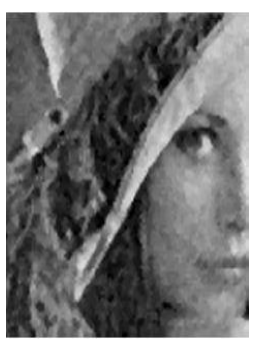

(E)

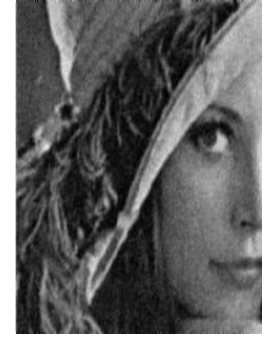

(G)
Fig. 8: Performance of various algorithms for Lena image. (A) Enlarged Original image. (B) Enlarged Corrupted image with 10 dB Gaussian noises. (C) PWMAD. (D) ROC. (E) SAWM. (F) SADA. (G) PA.

\section{CONCLUSION}

A Modified Spatially Adaptive Denoising Algorithm for an image corrupted by Gaussian noise is presented in this paper. By using the local statistics with in a selected window in a corrupted image, noise detection and noise removal filters are defined. The proposed algorithm is tested and compared with other existing algorithms such as SAWM, ROC, PWMAD, and SADA. From the comparison result it can be see that the proposed algorithm is better than the existing algorithms.

\section{REFERENCES}

[1] H. C. Andrew and B. R. Hunt. Digital Image Restoration. Prentice Hall, 1977.

[2] G. R. Arce. Nonlinear Signal Processing: A Statistical Approach. John Wiley and Sons Inc., 2007.
[3] T. A. Nodes and N. C. Gallagher. "Median filters: some modifications and their properties". IEEE Trans. Acoustics, Speech and Signal Processing, vol. 30, no.5, pp. 739-746, Oct. 1982.

[4] R. Yang, L. Lin, M. Gabbouj, J. Astola, and Y. Neuvo. "Optimal weighted median filters under structural constrains". IEEE Trans. Signal Processing, vol. 43, no.3, pp. 591-604, Mar. 1995.

[5] J. B. Bednar and T. K. Watt. "Alpha-trimmed means and their relationship to median filter". IEEE Trans. Acoustics, Speech and Signal Processing, vol. 32, no.1, pp. 145-153, Jan. 1984.

[6] Z. Wang and D. Zang. "Progressive switching median filter for removal of impulse noise from highly corrupted image". IEEE Trans. Circuits System II, vol. 46, no. 1, pp. 78-80, Jan. 1999.

[7] H. Ibrahim, Nicholas S. Kong, and Theam F. Ng. "Simple adaptive median filter for the removal of impulse noise from highly corrupted images". IEEE Trans. Consumer Electronics, vol. 54, no. 4, pp. 1920-1927, Nov. 2008.

[8] V. Crnojevic, V. Senk, and Z. Trpovski. "Advanced impulse detection based on pixel wise MAD". IEEE Signal process. Lett, vol. 11, no. 7, pp. 589-592, Jul. 2004.

[9] I. Aizenberg and C. Butakoff. "Effective impulse detector based on rank-order criteria". IEEE Signal process. Lett, vol. 11, no. 3, pp. 363-366, Mar. 2004.

[10] X. Zhang and Y. Xiong. "Impulse noise removal using directional differences based noise detector and adaptive weighted mean filter". IEEE Signal process. Lett, vol. 16, no. 4, pp. 295-298, Apr. 2009.

[11] T. A. Nguyen, W. S. Song, and M. C. Hong. "Spatially adaptive denoising algorithm for a single image corrupted by Gaussian noise". IEEE Trans. On Consumer Electronics, vol. 56, no. 3, pp. 1610-1625, Aug. 2010

\section{BIOGRAPHIES}

Arabinda Dash received B.Tech from Biju Patnaik

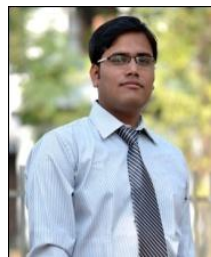
University of Technology, Rourkela, Odisha, India. He is currently pursuing M. Tech in Veer Surendra Sai University of Technology, Burla, Odisha, India. His research areas include Image Restoration and Image/Video Processing.

Sujaya Kumar Sathua received B. Tech from Veer

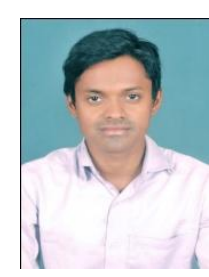
Surendra Sai University of Technology, Burla, Odisha, India. He took his M. Tech from National Institute of Technology, Rourkela, Odisha, India. Currently, he is working as an Assistant Professor at Veer Surendra Sai University of Technology, Burla, Odisha, India in the department of Information Technology. His research areas include Image Restoration, Image Processing and Algorithms. 